OPEN ACCESS

Edited by:

Tieliu Shi,

East China Normal University,

China

Reviewed by:

Dong Dong,

East China Normal University,

China

Abdallah El-Sayed Allam,

Tanta University, Egypt

*Correspondence

Yi LiU

liuyi-ly@126.com

Zhongtao Gai

gaizhongtao@sina.com

Specialty section:

This article was submitted to

Genetic Disorders,

a section of the journal

Frontiers in Genetics

Received: 29 June 2018

Accepted: 09 July 2019

Published: 22 August 2019

Citation:

Yan B, Wang $C$, Zhang $K$, Zhang $H$,

Gao M, Lv Y, Li X, Liu Y and Gai Z (2019) Novel Neonatal Variants of the

Carbamoyl Phosphate Synthetase 1

Deficiency: Two Case Reports and

Review of Literature.

Front. Genet. 10:718.

doi: 10.3389/fgene.2019.00718

\section{Novel Neonatal Variants of the Carbamoyl Phosphate Synthetase 1 Deficiency: Two Case Reports and Review of Literature}

\author{
Beibei Yan ${ }^{1}$, Chao Wang', Kaihui Zhang ${ }^{3}$, Haiyan Zhang ${ }^{3}$, Min $\mathrm{Gao}^{3}$, Yuqiang Lv', \\ Xiaoying $\mathrm{Li}^{1}$, Yi Liu ${ }^{3 *}$ and Zhongtao $\mathrm{Gai}^{3 *}$
}

${ }^{1}$ Neonatology Department, Qilu Children's Hospital of Shandong University, Ji'nan, China, ${ }^{2}$ Shandong Freshwater Fisheries Research Institute, Ji'nan, China, ${ }^{3}$ Pediatric Research Institute, Qilu Children's Hospital of Shandong University, Ji'nan, China

Carbamoyl phosphate synthetase I (CPS1) deficiency (CPS1D), is a rare autosomal recessive disorder, characterized by life-threatening hyperammonemia. In this study, we presented the detailed clinical features and genetic analysis of two patients with neonatal-onset CPS1D carrying two compound heterozygous variants of c.1631C > T (p.T544M)/c.1981G > T (p.G661C), and c.2896G > T (p.E966X)/c622-3C > G in CPS1 gene, individually. Out of them, three variants are novel, unreported including a missense (c.1981G > T, p.G661C), a nonsense (c.2896G > T, p.E966X), and a splicing change of c.622-3C > G. We reviewed all available publications regarding CPS1 mutations, and in total 264 different variants have been reported, with majority of 157 (59.5\%) missense, followed by 35 (13.2\%) small deletions. This study expanded the mutational spectrum of CPS1. Moreover, our cases and review further support the idea that most $(\geq 90 \%)$ of the mutations were "private" and only 10\% recurred in unrelated families.

Keywords: carbamoyl phosphate synthetase 1 deficiency, carbamoyl phosphate synthetase 1, urea cycle disorders, next-generation sequencing, missense, nonsense, deletion, splicing

\section{INTRODUCTION}

Carbamoyl phosphate synthetase I (CPS1) deficiency (CPS1D) is a rare autosomal recessive urea cycle disorder, characterized by hyperammonemia with the incidence of $1 / 50,000$ to $1 / 300,000$ (Díez-Fernández et al., 2015). CPS1D is currently divided into two types of neonatal onset and late onset, whereas CPS1D with severe manifestations of hyperammonemia is common in neonatalonset patients (Choi et al., 2017; Rokicki et al., 2017; Yang et al., 2017; Zhang et al., 2018). Typically, the neonatal-onset patient with CPS1D appears to be healthy at birth, but deteriorates rapidly into severe hyperammonemia, presenting poor feeding, vomiting, hypotonia, irritability, seizures, hypothermia, lethargy, coma, apnea, and even death after first feeding (Funghini et al., 2012; Choi et al., 2017; Rokicki et al., 2017; Zhang et al., 2018).

The function of urea cycle is to transform toxic ammonia into non-toxic urea. CPS1 catalyzes the initial and limiting step of the urea cycle, which is critical in the detoxification of excess ammonia, so CPS1D patient suffering from hyperammonemia will present a decreased level of citrulline but elevated glutamine in blood amino acid analysis, and a low level of orotic acid in urine test (Funghini et al., 2012; de Cima et al., 2015; Ali et al., 2016). 
It is difficult to timely diagnose CPS1D due to atypical manifestations like sudden onset, rapid progress, and low morbidity, as well as complicated and non-recurrent genetic mutations in CPS1 gene (Choi et al., 2017; Rokicki et al., 2017; Zhang and Li, 2017). For more than a decade, the diagnosis of CPS1D has been mainly relied on the laboratory tests of tandem mass spectrometry (MS/MS) including liquid chromatography-tandem mass spectrometry (LC-MS/MS) and gas chromatography mass spectrometry (GC/MS). MS/ MS is a high-throughput technique for measurement of the intermediate metabolites and has been widely used to distinguish dozens of metabolic diseases (Lehotay et al., 2011; Janecková et al., 2012; Hao et al., 2018). However, this technology cannot differentiate CPS1D from $N$-acetylglutamate synthase deficiency (NAGSD) in UCDs due to their similar intermediate metabolites. Until recently, next-generation sequencing (NGS), a powerful DNA sequencing technology, has revolutionized genomic research with great utility in the molecular diagnosis of genetic disorders (Choi et al., 2017; Jia et al., 2017; Rokicki et al., 2017; Li et al., 2018; Zhang et al., 2018), and has been proven reliable and important to detect CPS1 mutation for early diagnosis of CPS1D, as the severity of clinical manifestations in CPS1D patients is determined by the extent of CPS1 deficiency (Choi et al., 2017; Chen et al., 2018; Zhang et al., 2018).

In this study, we performed the clinical examinations and mutation analysis on two neonatal patients with CPS1D. The LC-MS/MS and GC/MS were carried out to detect amino acids in blood and organic acidurias in the urine, and then NGS was utilized to test the gene mutation. Strikingly, for the first time, we identified three novel pathogenic mutations of CPS1. To our knowledge, there have been so far only three reports of using NGS to detect CPS1 mutations for CPS1D diagnosis (Choi et al., 2017; Chen et al., 2018; Zhang et al., 2018). Our novel findings further expanded the mutational spectrum of CPS1 and demonstrated additional evidences of using NGS for precise identification of CPS1 mutations in patients.

\section{MATERIALS AND METHODS}

\section{Patients, Samples and Ethical Approval}

This study was approved by Medical Ethics Committee of Qilu Children's Hospital of Shandong University. The written informed consents were obtained from the parents of each study participant, and the patients' information was anonymized before submission. All the procedures performed in the study were in accordance with the Declaration of Helsinki.

Two patients from two unrelated families who were from the neonatal intensive care unit (NICU) of Qilu Children's Hospital of Shandong University (QCHSU) were firstly screened by LC/ MS-MS and GC/MS. The parents of both patients were healthy and non-consanguineous. Blood samples were obtained from the patients and their parents in accordance with informed consents in the study.

In addition, 100 blood samples from healthy children were collected as control samples for mutations validation.

\section{Routine Examination and Biochemical Laboratory Tests}

Routine physical examination, complete blood count (CBC), C-reactive protein (CRP), hemoculture, and biochemical laboratory tests, such as liver function, kidney function, glucose, ammonia, lactic acid, and blood gas analyses, were carried out.

The level of orotic acids in urine was measured by GC/MS with GCMS-QP2010 analyzer (Shimadzu, Tokyo, Japan) and analyzed by the Inborn Errors of Metabolism Screening System software (Shimadzu), whereas amino acids level in blood was detected by LC-MS/MS with Applied Biosystems API 3200 analyzer (ABSCIEX, Foster City, CA) and analyzed by the ChemoView software (ABSCIEX).

\section{Next-Generation Sequencing and Variant Discovery}

Genomic DNA was extracted and purified from peripheral blood of the two patients and their parents using TIANamp Blood Genomic DNA Purification Kit (Tiangen Biotech, Beijing, China). Whole-exome sequencing was applied to test mutation of genes in both patients. Approximately $3 \mu \mathrm{g}$ of genomic DNA was randomly fragmented. An exome enriched kit (Agilent, Santa Clara, CA) was used to obtain the coding exons and flanking intronic regions. The sequencing was performed using HiSeq2000 sequencer (Illumina, San Diego, CA). The obtained mean exome coverage was over $99.2 \%$, and average sequencing depth of each sample was $100 \%$. Raw data obtained from the sequencer were further analyzed including read alignment, variant calling, and annotation by SinoPath Enterprise Ltd (Beijing, China). Lowquality reads (quality score $\leq 20$ and sequencing depth $\leq 5$ ) in the raw data were removed. Filtered reads were aligned to the human reference genome (UCSC hg19 Feb.2009) using the BurrowsWheeler Aligner (Raney et al., 2014). Single-nucleotide variants (SNVs) and small insertions/deletions (indels) can be detected. Annotation was carried out by ANNOVAR for gene information, protein functional predictions, and population allele frequencies (Wang et al., 2010). Variants outside of coding regions and greater than $1 \%$ MAF (minor allele frequency) in the population were excluded.

\section{Bioinformatic Analysis and Verification of Mutations}

All known variants were reported according to the following databases: OMIM (http://www.ncbi.nlm.nih.gov/omim/limits), UCSC Genome Bioinformatics (http://genome.ucsc.edu/), Human Gene Mutation Database (http://www.hgmd.cf.ac.uk/ac/index. php), Single Nucleotide Polymorphism Database (dbSNP) (http:// www.ncbi.nlm.nih.gov/SNP/), 1000 Genomes Database (http:// browser.1000genomes.org), ExAC (http://exac.broadinstitute.org/ about), and gnomAD (http://gnomad.broadinstitute.org/). In silico analysis of the variants was carried out using PolyPhen-2 and SIFT and Mutation Taster to predict the pathogenicity. Human Splicing Finder (HSF) was applied to predict the effect of splicing variant. The multiple-sequence alignments were carried out by ClustalX. Modeling of affected protein structure was processed using 
SWISS-MODEL. The data analysis was conducted referring to the document (Jin et al., 2018). All the selected variants were classified as pathogenic, likely pathogenic, a variant of unknown significance (VUS), likely benign, or benign according to the American College of Medical Genetics and Genomics (ACMG) guidelines (Richards et al., 2015). The potential pathogenic mutations were validated by Sanger sequencing.

\section{RESULTS}

\section{Clinical Characteristics of Two Patients}

The clinical manifestations and laboratory data from the two patients were summarized in Table 1 . Both patients were neonatal-onset type presenting fulminant symptoms due to serious hyperammonemia so that the life-sustaining mechanical ventilation, medications of vasopressors, liquid infusion, and ammonia scavengers were administered.

Patient 1 (P1), a full-term female, the first child of healthy unrelated parents, was vaginally delivered. Her mother had regular prenatal care starting from 12 weeks of pregnancy. She was apparently healthy at birth with weight of $2.95 \mathrm{~kg}$, Apgar score of 10 at $1 \mathrm{~min}$ and $5 \mathrm{~min}$ after birth. The following day, however, she had a fever, and then gradually developed hyporeactiveness presenting respiratory distress, seizures, and acute circulatory collapse so she was immediately transported to NICU in QCHSU from local hospital. Laboratory tests revealed the abnormal blood indexes of ammonia, 1,404 $\mu \mathrm{mol} / \mathrm{L}$ (reference, 18-72 $\mu \mathrm{mol} / \mathrm{L}$ ); citrulline, $3.82 \mu \mathrm{mol} / \mathrm{L}$ (reference, 4-30 $\mu \mathrm{mol} / \mathrm{L}$ ); alanine, 1,264.4 $\mu \mathrm{mol} / \mathrm{L}$ (reference, $62.9-328 \mu \mathrm{mol} / \mathrm{L}$ ); lactic acid, $5.8 \mathrm{mmol} / \mathrm{L}$ (reference, $0.7-2.1 \mathrm{mmol} / \mathrm{L}$ ); glucose, $0.3 \mathrm{mmol} / \mathrm{L}$ (reference, 3.3-6.1 mmol/L); and white blood cells $22.06 \times 10^{9} / \mathrm{L}$ (reference, $5.0-14.5 \times 10^{9} / \mathrm{L}$ ), as well as abnormal urinary indexes of undetected orotic acid and elevated 3-MGA $15.7 \mathrm{mmol} / \mathrm{L}$ (ref. 0-4 $\mathrm{mmol} / \mathrm{L}$ ). The chest radiograph result reported pneumonia and possible atelectasis. Patient heart rate reached up to 180 beats/min but the ejection fraction was only $38 \%$ and no signs of congenital heart disease (Table 1).

The mechanical ventilation and medications of vasopressors, liquid infusion, and antibiotics, ammonia scavengers, such as dopamine, dobutamine, dilator, meropenem, lactulose, and L-arginine were administered immediately. Meanwhile, oral feeding was forbidden, and total parenteral nutrition with lower amino acid was administered. Unfortunately, the patient deteriorated continually into multiple-organ failure and even had cardiac arrest with no spontaneous breathing. Considering the poor prognosis, her parents gave up her treatment, and she died at age of 5 days.

Patient 2 (P2), a full-term girl, the second child of healthy unrelated parents, was vaginally delivered. The first child of the family died suddenly at the third day after birth without a definite diagnosis. Her mother had regular prenatal care, and she was normal at birth with a weight of $2.9 \mathrm{~kg}$, Apgar score of 10 at 1 and $5 \mathrm{~min}$ after birth. On the third day, however, she had a sudden onset of hyperlactacidemia and deteriorated even faster than P1 did. At the beginning, she was hyporeactive presenting grunting and anorectic, but no manifestations of
TABLE 1 | Clinical and laboratory data of the two patients with CPS1D.

\begin{tabular}{|c|c|c|}
\hline Patients & P1 & P2 \\
\hline Gender & Female & Female \\
\hline Age at onset & $2 \mathrm{D}$ & $3 D$ \\
\hline Age deceased & $5 \mathrm{D}$ & 4D \\
\hline Complications during pregnancy & Uneventful & uneventful \\
\hline Weeks gestation at delivery & 40 & $39+5$ \\
\hline Birth weight (kg), Apgar score & $2.95,10$ & $2.9,10$ \\
\hline Clinical course & fulminant & fulminant \\
\hline \multicolumn{3}{|l|}{ Clinical features } \\
\hline Fever & + & - \\
\hline Seizure & + & - \\
\hline Coma & + & + \\
\hline Cyanosis & + & + \\
\hline Breathing & weak & grunting \\
\hline Hemorrhage & - & Lung, stomach \\
\hline Cardiac failure & + & + \\
\hline Poor feeding & + & + \\
\hline Abdominal distention & & + \\
\hline Urine & oliguria & anuria \\
\hline \multicolumn{3}{|l|}{ Arterial blood gas analysis } \\
\hline $\mathrm{PH}$ (reference, 7.25-7.45) & $7.13 \downarrow$ & $7.10 \downarrow$ \\
\hline $\mathrm{PO}_{2}$ (reference, $50-80 \mathrm{~mm} \mathrm{Hg}$ ) & 67 & 72 \\
\hline $\mathrm{PCO}_{2}$ (reference, $40-60 \mathrm{~mm} \mathrm{Hg}$ ) & 42 & $62 \uparrow$ \\
\hline $\mathrm{HCO}_{3}$ (reference, 19-30 mmol/L) & $12 \downarrow$ & 19.2 \\
\hline $\mathrm{BE}$ (reference, -3 to +3 ) & $-18 \downarrow$ & $-10.5 \downarrow$ \\
\hline \multicolumn{3}{|l|}{ Blood routine test } \\
\hline Red blood cells (reference, 3.5-5.5×1012/L) & $5.68 \uparrow$ & $3.35 \downarrow$ \\
\hline White blood cells (reference, 5.0-14.5×109/L) & $22.06 \uparrow$ & 24.77 \\
\hline Platelet count (reference, 100-300×109/L) & $354 \uparrow$ & 290 \\
\hline Hemoglobin (reference, 138-218 g/L) & 195 & $117 \downarrow$ \\
\hline \multicolumn{3}{|l|}{ Urine routine test } \\
\hline BLD (negative) & ++ & +++ \\
\hline PRO (negative) & ++ & + \\
\hline KET (negative) & - & - \\
\hline \multicolumn{3}{|l|}{ Blood biochemical tests } \\
\hline ALT (reference, 0-38 U/L) & 37 & 24 \\
\hline Lactic acid (reference, 0.7-2.1 mmol/L) & $5.8 \uparrow$ & $5.6 \uparrow$ \\
\hline Glucose (reference, 3.3-6.1 mmol/L) & $0.3 \downarrow$ & $12 \uparrow$ \\
\hline Potassium (reference, 3.5-5.5 mmol/L) & $7.4 \uparrow$ & 4.6 \\
\hline Sodium (reference, $135-145$ mmol/L) & $154 \uparrow$ & 142 \\
\hline AST (reference, 0-38 U/L) & $130 \uparrow$ & $78 \uparrow$ \\
\hline CK (reference, 21-220 U/L) & $936 \uparrow$ & $780 \uparrow$ \\
\hline CK-MB (reference, 0-25 U/L) & $30 \uparrow$ & $67 \uparrow$ \\
\hline PCT (reference, $\leq 0.5$ ng/ml) & $67.322 \uparrow$ & $0.794 \uparrow$ \\
\hline ammonia (reference, $18-72 \mu \mathrm{mol} / \mathrm{L}$ ) & $1404 \uparrow$ & $823 \uparrow$ \\
\hline \multicolumn{3}{|l|}{ Blood mass spectrometry profile } \\
\hline Citrulline (reference, $4-30 \mu \mathrm{mol} / \mathrm{L}$ ) & $3.82 \downarrow$ & $3.08 \downarrow$ \\
\hline Alanine (reference, 62.9-328 $\mu \mathrm{mol} / \mathrm{L})$ & $1264.4 \uparrow$ & $3337.99 \uparrow$ \\
\hline Proline (reference, $72-293 \mu \mathrm{mol} / \mathrm{L}$ ) & $634.45 \uparrow$ & $413.38 \uparrow$ \\
\hline Ornithine (reference, 42-358 $\mu \mathrm{mol} / \mathrm{L}$ ) & 69.01 & 106.95 \\
\hline \multicolumn{3}{|l|}{ Urinary organic acids } \\
\hline Urinary orotic acid (reference, 0-2 mmol/L) & 0 & 0 \\
\hline Urinary 3-MGA (reference, 0-4 mmol/L) & $15.7 \uparrow$ & $45.75 \uparrow$ \\
\hline \multicolumn{3}{|l|}{ Chest X-ray } \\
\hline Pneumonia & + & - \\
\hline Pneumorrhagia & - & + \\
\hline \multicolumn{3}{|l|}{ Echocardiography } \\
\hline Ejection fraction & $38 \% \downarrow$ & $37 \% \downarrow$ \\
\hline Patent ductus arteriosus & + & - \\
\hline Patent foramen ovale & + & + \\
\hline \multicolumn{3}{|l|}{ CPS1 sequencing } \\
\hline Allele 1 (from father) & $\begin{array}{l}\text { c. } 1631 \mathrm{C}>\mathrm{T} \\
\text { (p.T544M) }\end{array}$ & $\begin{array}{l}\text { c.2896G > T } \\
\text { (p.E966X) }\end{array}$ \\
\hline Allele 2 (from mother) & $\begin{array}{l}\text { c.1981G > T } \\
\text { (p.G661C) }\end{array}$ & c. $622-3 \mathrm{C}>\mathrm{G}$ \\
\hline
\end{tabular}

+positive, $\uparrow$ elevated, $\downarrow d e c r e a s e d$. 
fever, vomiting, and seizures. Five hours later, she developed pneumorrhagia, gastrointestinal hemorrhage, and anuria, so that she was immediately transferred to NICU in QCHSU from her local hospital. On the way to the hospital, her heart rate and oxygen saturation could not be maintained; cardio-pulmonary resuscitation and mechanical ventilator had to be administered. Nevertheless, she deteriorated very quickly, presenting coma, shock, and irregular respirations. When she was admitted, she looked pale with reduced perfusion and a low ejection fraction $(37.9 \%)$ in her echocardiography. The blood flowed out of her intratracheal tube and nose. Her pupil diameter was about $4 \mathrm{~mm}$, and pupillary reflex disappeared. Laboratory tests revealed abnormal blood indexes of ammonia, $823 \mu \mathrm{mol} / \mathrm{L}$; citrulline, $3.08 \mathrm{mmol} / \mathrm{L}$; alanine, 3,337.99 $\mu \mathrm{mol} / \mathrm{L}$ (reference, 62.9-328 $\mu \mathrm{mol} / \mathrm{L}$ ); lactic acid, $5.6 \mathrm{mmol} / \mathrm{L}$; glucose, $12 \mathrm{mmol} / \mathrm{L}$; and white blood cells, $24.77 \times 10^{9} / \mathrm{L}$, as well as abnormal urinary indexes of undetected orotic acid and increases 3-MGA $45.75 \mathrm{mmol} / \mathrm{L}$. The chest radiograph showed exudative lesions, which matched her pulmonary hemorrhage.

This patient received immediate treatment that was similar as P1 with mechanical ventilation, vasopressors, liquid infusion, ammonia scavengers (such as lactulose and L-arginine), dopamine, dobutamine, dilator, as well as total parenteral nutrition with lower amino acid. She continued to deteriorate with tremendous speed and no sign of improvement after $13 \mathrm{~h}$ of admission and died at the age of 4 days.

\section{Genetic Analysis and Pathogenicity Prediction}

Whole-exome sequencing showed two compound heterozygous variants of the CPS1 gene in both $\mathrm{P} 1$ and $\mathrm{P} 2$, individually, including two missense variants of c.1631C > T (p.T544M) and c.1981G > T (p.G661C) found in P1, a nonsense variant c.2896G > T (p.E966X) and a splicing variant c.622-3C > G detected in P2. Of which, the variant c.1631C > T (p.T544M) was a known pathogenic mutation causing CPS1D (Finckh et al., 1998; Häberle et al., 2011) (Table 1 and Figures 1A, B), whereas the remaining three variants of c.1981G > T (p.G661C), c.622-3C > G, and c.2896G > T (p.G966X) were novel and unreported in publications and public databases of OMIM, UCSC, HGMD, dbSNP, 1000 genomes, ExAC, and gnomAD. The missense c.1981G > T (p.G661C) occurred with an amino acid change from a nonpolar amino acid of glycine $(G)$ to a polar amino acid of cysteine (C); the nonsense c.2896G > T (p.G966X) would create a premature stop codon; the splicing change c.622-3C > G was predicated to affect acceptor splice site. There were no mutations found in the control samples by using Sanger sequencing.

The pathogenicity of three novel variants was further analyzed using various prediction online tools. In brief, HSF was applied to assess the potential impacts on the splicing of three novel variants as all these variants located near intron-exon junction. The predicted results showed that all three variants in the exon 17 (c.1981G > T), intron 7 (c.622-3C > G), and exon 24 (c.2896G > T) probably affect the splice sites (Figures 2A-C). The missense mutations of c.1981G > T (p.G661C) were predicted to be pathogenic by SIFT, MutationTaster, and PolyPhen-2 (Figure 3A). The conservation analysis of the variants of c.1981G > T (p.G661C) and c.2896G > T (p.G966X) in CPS1 showed that both sites were highly conservative in different species by using ClustalX (Figures 3B, C), whereas the missense variant c.1981G > T (p.G661C) was predicted to change the highly evolutionary conserved amino acid in CPS1, and the nonsense variant c.2896G > T (p.G966X) causing a premature stop could generate a truncated protein with missing conserved site of CPS1. In addition, the CPS1 protein crystallographic structure of both mutant types (p.G661C and p.G966X) revealed the changes of side strand structure and $\mathrm{H}$-bond in variant of p.G661C, and a truncated protein with loss of 534 amino acids in variant of p.G966X (Figures 4A, B). All the mutation information and clinical data were uploaded into eRAM (Jia et al., 2018).

\section{DISCUSSION}

Pediatric rare diseases are often rapid deterioration with high mortality, which can be obviously improved by early diagnosis and treatment (Ni et al., 2017). CPS1D is a rare inborn error of UCD caused by CPS1 deficiency manifesting sudden onset, rapid progress, and low morbidity. In this study, we presented the detailed clinical manifestations and mutation analysis of two neonatal CPS1D cases. First, the blood ammonia, amino acids, and urine organic acids test results reported severe hyperammonemia on both patients, and the patients have very high levels of alanine and decreased levels of citrulline in blood, as well as the increased levels of 3-MGA and decreased levels of orotic acid in urine. We therefore referred patients for whole-exome sequencing to determine the genetic cause of this inborn error of metabolism. After validation of Sanger sequencing, two compound heterozygous variants in CPS1 were identified in both patients, and one missense variant (c.1631C > T, p.T544M) was of known pathogenicity (Finchh et al., 1998; Häberle et al., 2011), whereas other three were novel and predicted to be pathogenic. Therefore, both patients were finally diagnosed as neonatal-onset CPS1D caused by CPS1 mutations. To our knowledge, this study is the fifth case report of CPS1D in China and the 262-264th novel mutations in CPS1 documented in the world (Chen et al., 2013; Yang et al., 2017; Chen et al., 2018; Zhang et al., 2018), which expands the mutation spectrum of CPS1 (Supplementary Tables S1 to S4).

CPS1 is an enzyme that catalyzes the first and ratelimiting reaction of three steps in ammonia detoxification of the urea cycle from ammonia to carbamoyl phosphate (de Cima et al., 2015; Ali et al., 2016). Normal function of the urea cycle requires six enzymes, including CPS1 as well as two mitochondrial transporters (Helman et al., 2014). CPS1 deficiency caused by CPS1 gene mutation usually leads to accumulation of ammonia in the blood and thereby presents severe hyperammonemia, which is neurotoxic resulting in neonatal death or severe and irreversible brain damage in the developing and mature brain (Funghini et al., 2012; Choi 


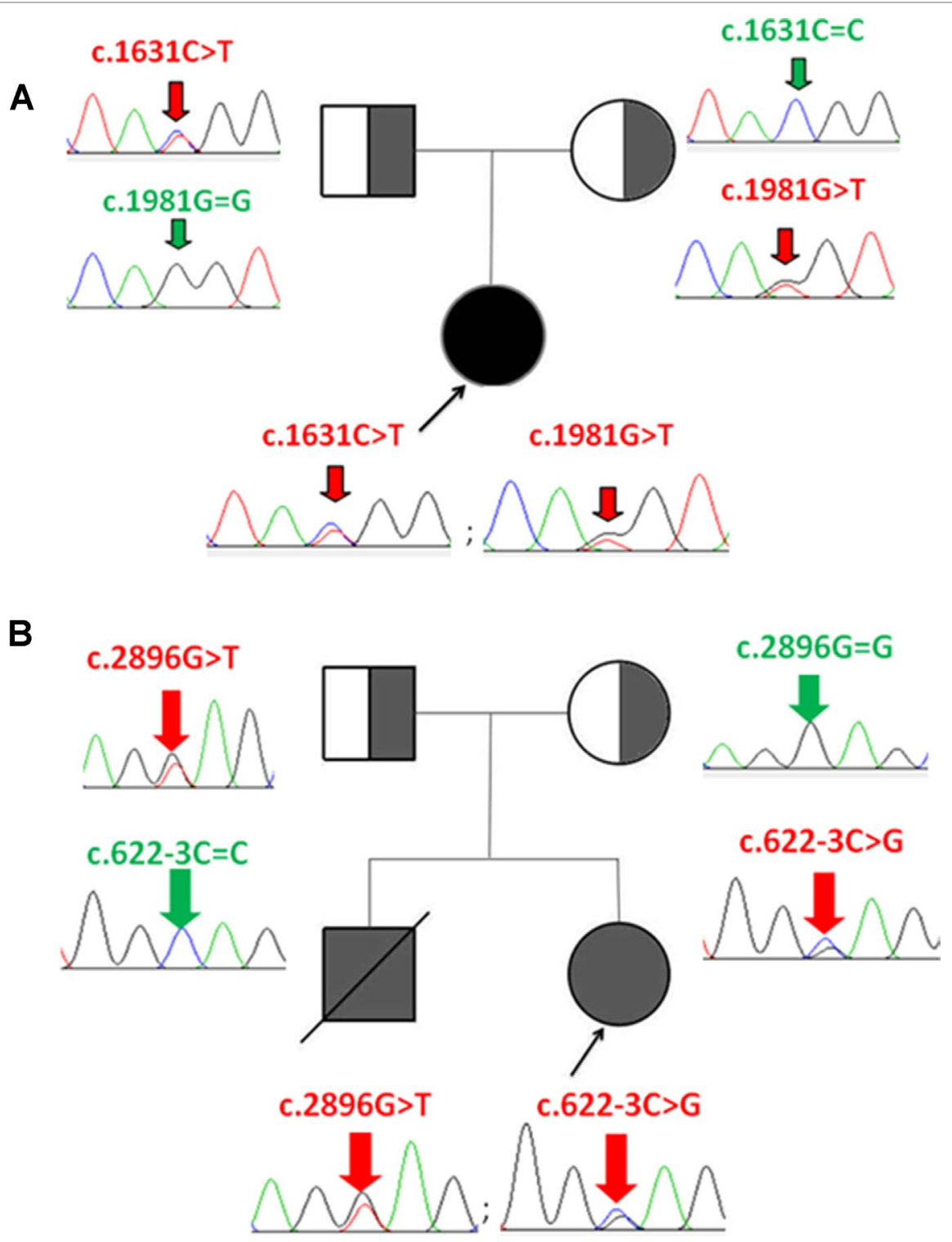

FIGURE 1 | Identification of CPS1 mutations and pedigree of the two families with CPS1D probands. (A) P1 has two compound heterozygous mutations of C.1631C > T and C.1981G > T, inherited from her father and mother, respectively. (B) P2 has two compound heterozygous mutations of c.2896G > T and c.622-3C > G, inherited from her father and mother, respectively.

et al., 2017; Rokicki et al., 2017; Yang et al., 2017). CPS1D are divided into two types of lethal neonatal-onset or less severe late-onset based on the age of onset, clinical features, and severity of CPS1 deficiency (Diez-Fernandez et al., 2017). To date, most of the reported CPS1D cases are neonatal-onset with severe hyperammonemia and usually died of multiorgan failure. Signs and symptoms in patients with CPS1D are often atypical with rapid progression and extremely low morbidity, which makes the clinical diagnosis difficult (Funghini et al., 2012; Choi et al., 2017; Rokicki et al., 2017).

As a result, the diagnosis of CPS1D is heavily dependent on laboratory data, such as blood ammonia, blood amino acids, urine organic acids, and genetic testing. The determination of blood ammonia concentration is critical for early clinical evaluation as it often reaches $150 \mu \mathrm{mol} / \mathrm{L}$ or higher in acute stage (László et al., 1991). Abnormal level of amino acids can be detected by mass spectrometry, like elevated blood glutamate, glutamine, and alanine, and reduced citrulline and arginine, whereas decreased urinary orotic acid and increased urinary 3-MGA (Lehotay et al., 2011; Janecková et al., 2012). Next-generation sequencing has been increasingly accessible in clinical laboratory for precise diagnosis of inborn errors of metabolism, including CPS1D (Choi et al., 2017; Yang et al., 2017; Li et al., 2018); for the biochemical tests mentioned above, one cannot distinguish from different types of UCD, particularly $\mathrm{N}$-acetylglutamate synthase deficiency (NAGSD) from CPS1D due to similar intermediary metabolites (Choi et al., 2017). 
A

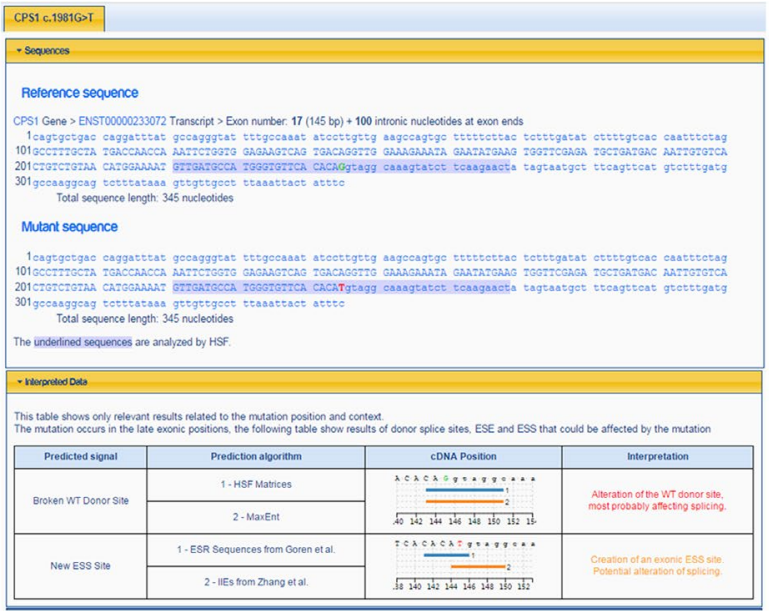

B

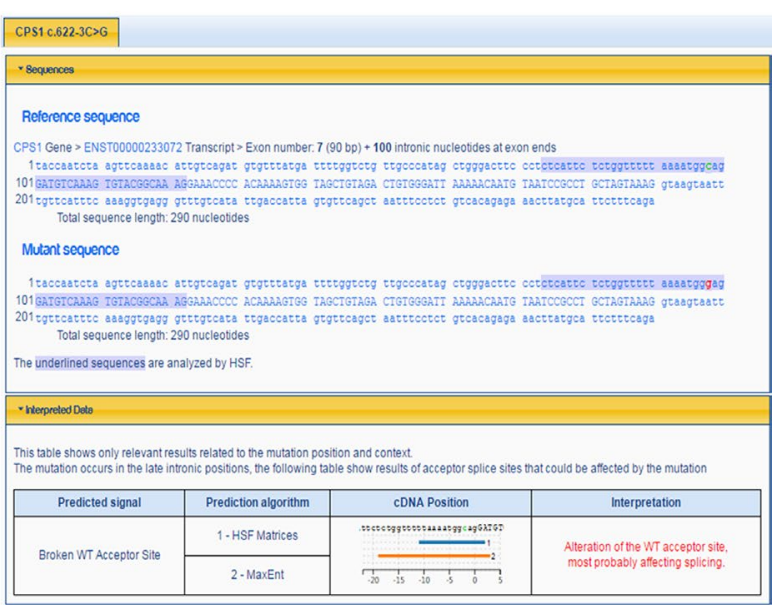

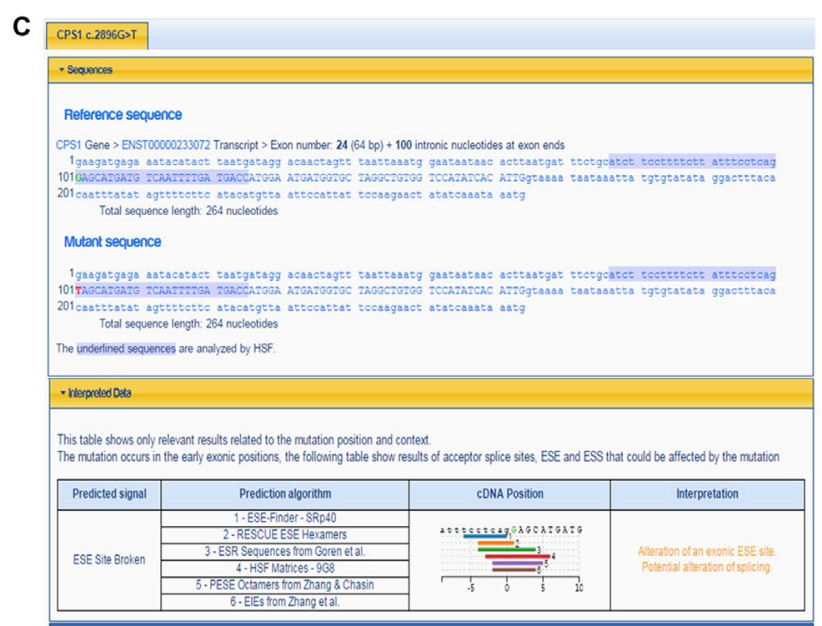

FIGURE 2 | Prediction of splicing errors for three novel mutations of CPS1 by HSF. (A) Prediction of splicing errors for C.1981G > T of CPS1 shows either alteration of the donor site of CPS1 affecting splicing or create an exonic ESS (exonic splicing silencer) site, potentially affecting splicing. (B) Prediction of splicing errors for c.622-3C > G of CPS1 shows the alteration of the acceptor site of CPS1 affecting splicing. (C) Prediction of splicing errors for c.2896G > T of CPS1 shows alteration of an exonic ESE (exonic splicing enhancer) site, potentially affecting splicing.

CPS1 (NM_001875.4) located on chromosome 2q34, spans over $122 \mathrm{~kb}$ consisting of 38 exons, which encode a polypeptide of 1,500 amino acids. Up to now, different variations of CPS1 have been reported, including missense, nonsense, small deletions, small insertions, small indels (insertions+deletions), and large deletions. As far as both cases of this study are concerned, two missense variants of c.1631C > T (p.T544M) and c.1981G > T (p.G661C) were found in a 2-day neonate girl (P1) with severe hyperammonemia. The variant of c.1631C > T (p.T544M) was a previously reported mutation (Finckh et al., 1998; Häberle et al., 2011) and was proven in the expression study causing large decrease of the enzyme activity due to hampering of the cross-talk between the bicarbonate phosphorylation domain (BPSD) and the allosteric NAG binding domain (ASD) (Diez-Fernandez et al., 2013). Another missense mutation (c.1981G > T, p.G661C) in P1 was novel and unreported. The pathogenicity of c.1981G > T (p.G661C) was analyzed and predicted as "deleterious" by SIFT, "protein features affected" by MutationTaster, and "probably damaging" with a score of 1.000 (sensitivity: 0.00; specificity: 1.00) by PolyPhen-2 (Figure 3A). As the variant occurred at the last exon base of the 17 exon, the potential splicing effect was assessed by HSF, which revealed a possible donor site error affecting the splicing of mRNA (Figure 2A). Moreover, the amino acid substitution site was highly conservative in different species analyzed by ClustalX (Figure 3B), so the alteration of p.G661C from glycine to cysteine was predicted to not only interrupt the conserved position of glycine but also change the side strand structure in the CPS1 protein crystallographic model, which produced a defective protein (Figure 4A). In addition, the mutation site of c.1981G > T (p.G661C) is identical as that of a known variant c.1981G > C (p.G661R) with the substitution of a small glycine residue by a large side chain, which was predicted to decrease structural stability of the protein (Funghini et al., 2012). Thus, we inferred that the substitution of amino acids from glycine to cysteine (p.G661C) might have a similar damage to the CPS1 
A

\section{p.G661C}

This mutation is predicted to be PROBABLY DAMAGING with a score of 1.000 (sensitivity: 0.00 ; specificity: 1.00 )

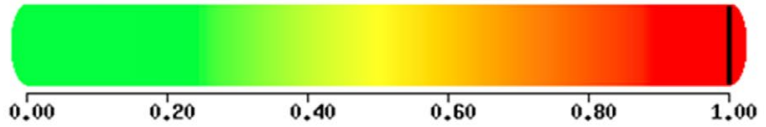

B

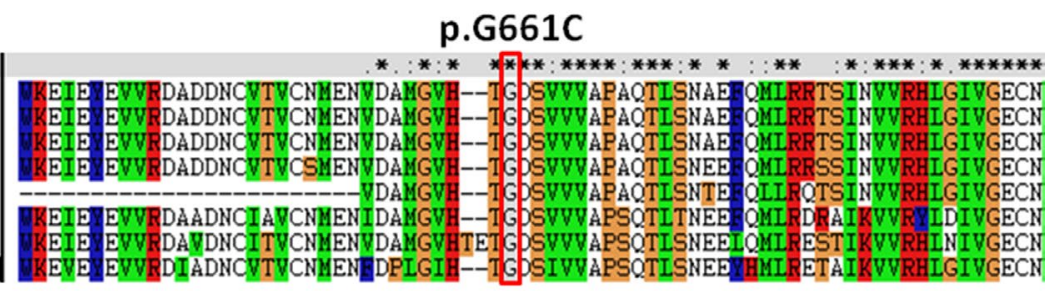

C

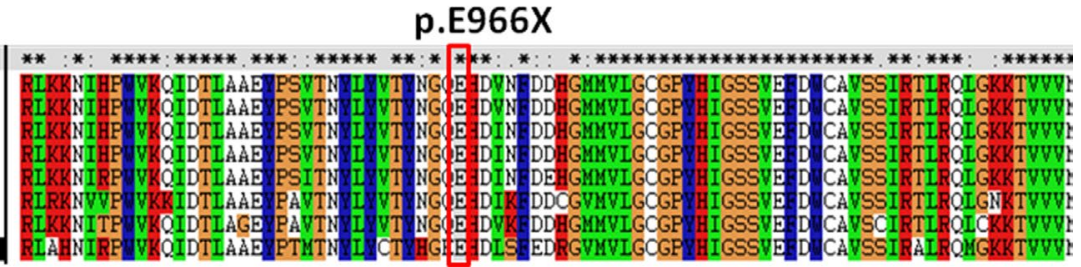

FIGURE 3 | Pathogenicity and conservation analysis of the missense and nonsense mutations of CPS1. (A) Pathogenicity analysis of c.1981G > T (p.G661C) shows "probably damaging" with a score of 1.000 (sensitivity: 0.00; specificity: 1.00) by PolyPhen-2. (B) In silico analysis of c.1981G > T (p.G661C) in CPS1 shows the site p.G661 highly conservative in different species of human, chimpanzee, bushbaby, horse, alpaca, chicken, anole, and cod. (C) In silico analysis of c.2896G > T (p.E966X) in CPS1 shows the site p.E966 highly conservative in different species of human, chimpanzee, bushbaby, horse, alpaca, chicken, anole, and cod.

stability, and both missense mutations in CPS1, leading to defects of enzyme function, are therefore the genetic cause of the patient with CPS1D.

A nonsense variant of c.2896G > T (p.G966X) and a splicing site change of c.622-3C > G were detected in a 3-day neonatal girl (P2). Since the nonsense variant site (c.2896G > T, p.G966X) is the first exon base of the 24th exon, its probable impact on splicing was assessed by HSF and revealed a potential effect of splicing with the alteration of an ESE (exonic splicing enhancer) site (Figure 2C). Besides, this position of glycine was highly conservative in different species analyzed by ClustalX (Figure 3C); the variant was predicted to generate a truncated protein with a loss of 534 amino acids and abolish the activity of the enzyme. The crystallographic structure model of the G966X further demonstrated the truncated protein (Figure 4B). The mutation of c.622-3C > G in intron 7 was a splicing site change that was predicted to alter the acceptor site of CPS1 gene and affect mRNA splicing, which would produce a nonfunctional enzyme (Figure 2B). The severe phenotype of $\mathrm{P} 2$ with more rapid progress to multiple-organ failure within $13 \mathrm{~h}$ from her onset suggested that both alleles encode a nonfunctional protein. Additionally, an unusual death of the first boy in the family drew our attention. The first child was born at term and apparently healthy after a normal pregnancy. He had sudden deterioration and died on the third day without a definite diagnosis. The retrospective analysis of the first boy from this family demonstrated that he had similar features as P2. We conjectured that the first child might carry the identically compound heterozygous variants inherited from their father and mother as his sister P2. On this occasion, genetic counseling and prenatal genetic testing are necessary for the subsequent pregnancy. Considering the positions of the novel mutations and their potential splicing defects, RT-PCR should be used to identify the possibly aberrant transcripts; unfortunately, we failed to get the RNA from both patients due to their parents' refusal.

We reviewed all publications of CPS1 variants in cases of CPS1D, and a total of 264 CPS1 different variations (including the 3 variants in this study) have been reported. Among them, the missense variants were the majority accounting for 157 (59.5\%), followed by small deletions of 35 (13.2\%), splice site changes of $25(9.5 \%)$, and nonsense of $22(8.3 \%)$, whereas the minority of the variants were $4(1.5 \%)$ small indels and $5(1.9 \%)$ large deletions with missing 1,000 bp to 767 $\mathrm{kb}$, which were detected by genomic microarray (Figure 5). Of the variants, $81(30.7 \%)$ were predicted to cause protein truncation, including 22 nonsense, 31 small deletions, 16 small insertions, 4 small indels, 6 splicing site changes, and 2 large deletions. Our reviewing data further clarified that most CPS1 variants $(\geq 90 \%)$ were "private" with non-recurrence, and the few recurrent mutations tended to occur at CpG dinucleotides, which made the diagnosis more complicated (Supplementary Tables S1 to S4) (Hoshide et al., 1993; Finckh et al., 1998; Summar et al., 1998; Ihara et al., 1999; Aoshima et al., 2001a; Aoshima et al., 2001b; Rapp et al., 2001; Wakutani et al., 2001; Häberle et al., 2003; Eeds et al., 2006; Kurokawa et al., 2007; 
A

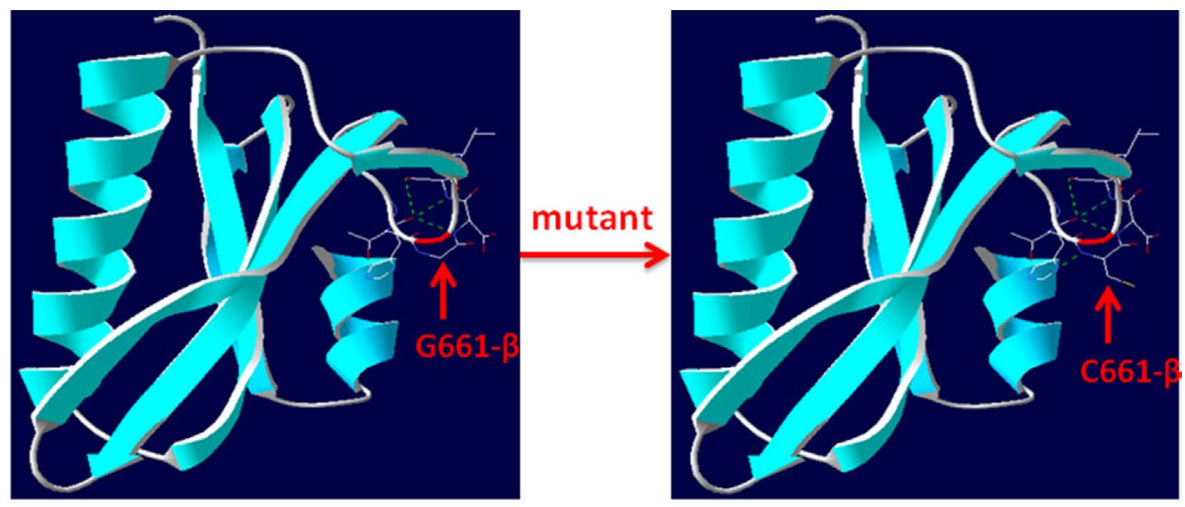

B

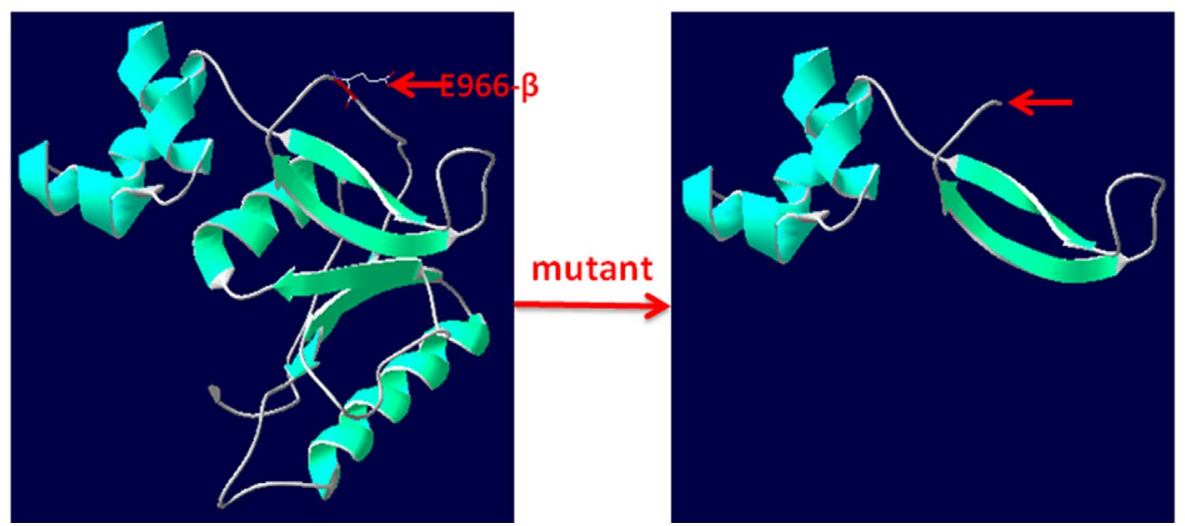

FIGURE 4 | 3D structure of wild type and mutant type of p.G661C and p.E966X in CPS1. (A) Wild type and mutant type of p.G661 in CPS1 shows the mutation of G661C changes the side strand structure and H-bond. (B) Wild type and mutant type of E966 in CPS1 shows the mutation of E661X leading to a truncated protein with missing 534 amino acids. The red arrows marked the sites of wild-type G661, E966, and mutated C661, X966.

\section{Distribution of mutation spectrum in CPS1}

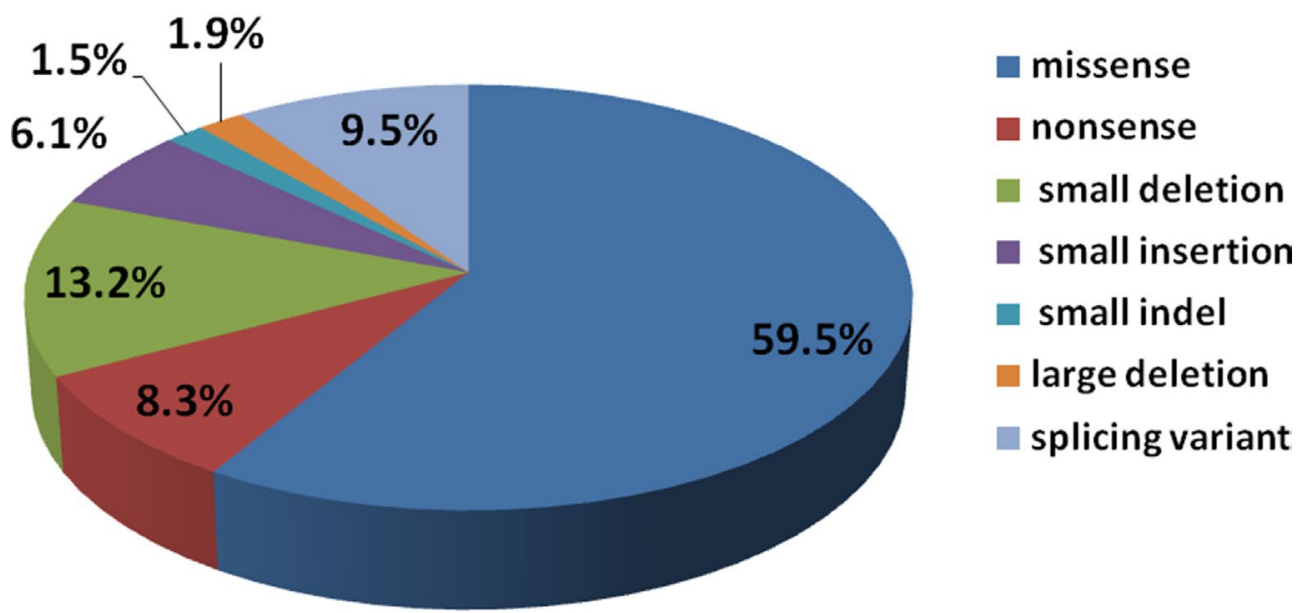

FIGURE 5 | Distribution of mutation spectrum in CPS1 reported in both cases and literature. A total of 259 mutations in CPS1 include $58.7 \%$ missense, $8.5 \%$ nonsense, $13.5 \%$ small deletion, $6.2 \%$ small insertion, $9.6 \%$ splicing, $1.6 \%$ small indel, and $1.9 \%$ large deletion. 
Khayat, 2009; Ono et al., 2009; Pekkala et al., 2010; Häberle et al., 2011; Wang et al., 2011; Funghini et al., 2012; Kretz et al., 2012; Diez-Fernandez et al., 2014; Ali et al., 2016; Choi et al., 2017; Rokicki et al., 2017; Yang et al., 2017; Chen et al., 2018; Zhang et al., 2018).

Encoded by CPS1 gene, CPS1 is a complex multidomain enzyme, composed of a $40-\mathrm{kDa} \mathrm{N}$-terminal moiety with two unknown function domains and a $120-\mathrm{kDa}$ C-terminal moiety involving four domains of bicarbonate phosphorylation (BPSD), integrating (ID), carbamate phosphorylation (CPSD), and allosteric NAG binding (ASD) (Diez-Fernandez et al., 2014; de Cima et al., 2015). The C-terminal moiety contains two ATP-binding sites, catalyzing the synthesis of carbamoyl phosphate from bicarbonate, ATP, and ammonia and has been discovered to possess missense mutations of CPS1 with high frequency and plays a critical integrating role in folding of structural elements leading to decreased yield of CPS1 (Figure 6) (Häberle et al., 2011; Diez-Fernandez et al., 2013; Diez-Fernandez et al., 2014). We analyzed the distribution of the 264 mutations and found that 66 (25\%) variants were located at $\mathrm{N}$-terminal moiety, whereas 198 (75\%) mutations were at C-terminal moiety involving 76 variants at BPSD, 29 at ID, 75 at CPSD, and 18 at ASD.
Three novel mutations were found in the study, two located at BPSD of C-terminal and one at $\mathrm{N}$-terminal moiety, which further supported the importance of the C-terminal moiety in maintaining the function of CPS1.

At present, the treatment of CPS1D is to strictly follow the recommendations of UCDs, which focuses on reduction of ammonia production by a restricted protein diet and management of ammonia scavengers, such as sodium benzoate, sodium phenylbutyrate, and sodium phenylacetate, as well as drugs of $\mathrm{L}$-arginine and L-citrulline to improve the residual urea cycle function and the renal excretion of ammonia (Diez-Fernandez and Häberle, 2017). In case of severe hyperammonemia, hemodialysis or peritoneal dialysis can be administered (Häberle, 2012; Diez-Fernandez and Häberle, 2017). However, these approaches cannot cure CPS1D, and the only available cure currently is liver transplantation, which has demonstrated excellent results with approximately 90\% survival rate in UCD children, though it is limited by donor sources (Diez-Fernandez and Häberle, 2017; Zhang and Li, 2017). To date, most of the CPS1D patients died before receiving the confirmed diagnosis, so the detection of blood ammonia, blood amino acids, urine organic acid, and next-generation sequencing should be performed as early as possible.

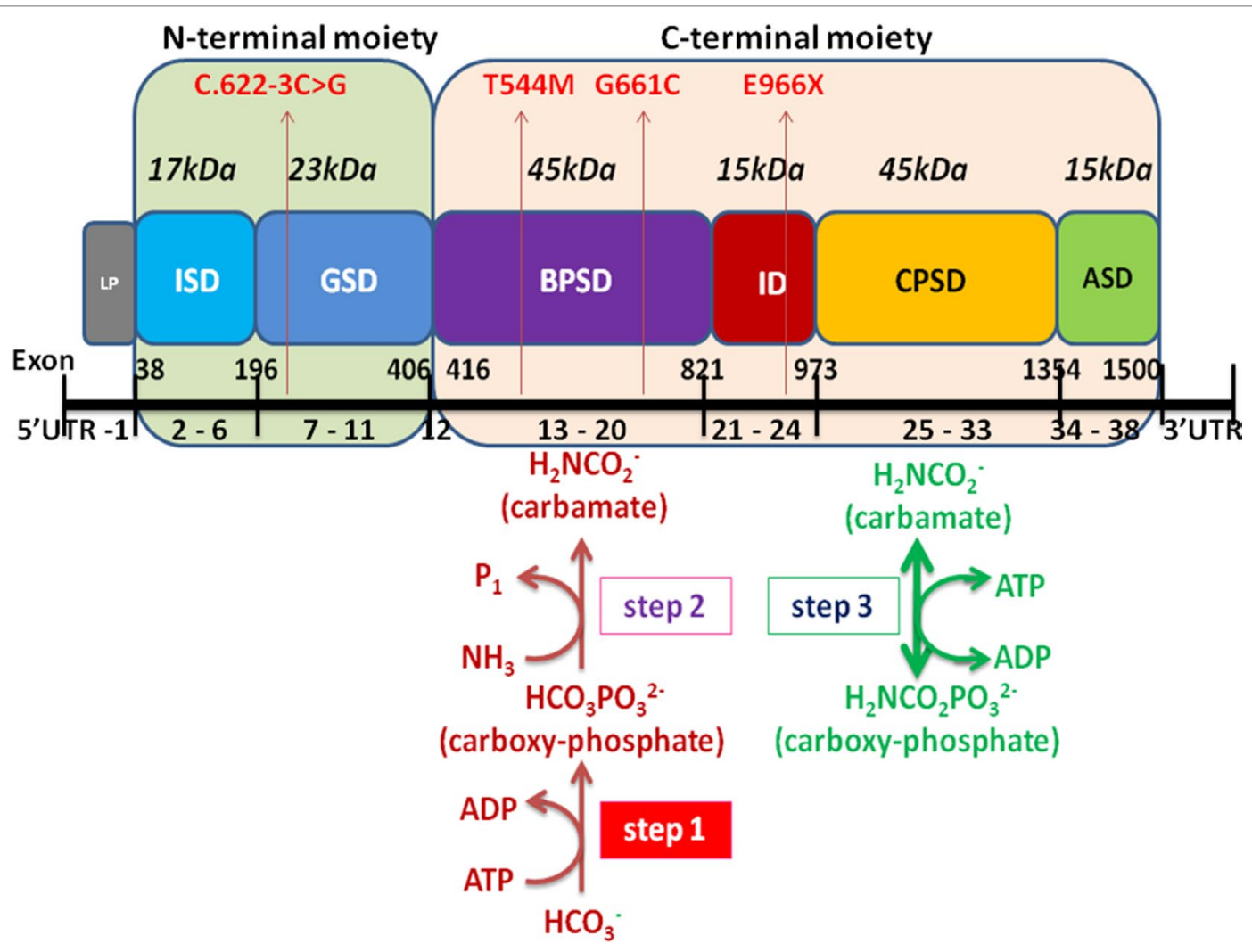

FIGURE 6 | Schematic representation of the CPS1 domain and exons modified from Diez-Fernandez et al. (2014) and Ali et al. (2016). The CPS1 polypeptide consists of the 40-kDa N-terminal moiety and 120-kDa C-terminal human CPS1 domain that correspond to small and large subunits of E. coli CPS, respectively. The different color boxes represent the different domains of CPS1. LP mitochondrial targeting peptide is not present in mature CPS1. ISD, inter-subunit domain; GSD, ancestral inactive glutaminase; BPSD, bicarbonate phosphorylation; ID, integrating domain; CPSD, carbamate phosphorylation; ASD NAG binding domain. The black line at the bottom represents the exons of CPS1, including 5'UTR, exon 1-38, and 3'UTR. Four mutations of CPS1 in this study are shown in red arrow. 


\section{CONCLUSION}

In this study, we presented the detailed clinical features and genetic analysis of two patients with neonatal-onset CPS1D and discovered three novel pathogenic variants in CPS 1 by whole-exome sequencing with a comprehensive outline of available publications regarding CPS1 gene mutations. A total 264 different variants of CPS1 have been reported with the majority of 157 (59.5\%) missense, followed by small deletions of $35(13.2 \%)$, and the minority of $5(1.9 \%)$ large deletions and $4(1.5 \%)$ indels, of which $81(30.7 \%)$ were predicted to cause protein truncation. Our data further expand the spectrum of CPS1 mutation and support the clinical applicability of wholeexome sequencing for genetic diagnosis of UCD.

\section{ETHICS STATEMENT}

The work was approved by Medical Ethics Committee of Qilu Children's Hospital of Shandong University. Written informed consents was obtained from the patients' parents and the patients' information was anonymized before submission. All the procedures performed in the study were in accordance with the Declaration of Helsinki.

\section{REFERENCES}

Ali, E. Z., Khalid, M. K. N. M., Yunus, Z. M., Yakob, Y., Chin, C. B., Latif, K. A., et al. (2016). Carbamoyl phosphate synthetase 1 (CPS1) deficiency: clinical, biochemical, and molecular characterization in Malaysian patients. Eur. J. Pediatr. 175 (3), 339-346. doi: 10.1007/s00431-015-2644-Z

Aoshima, T., Kajita, M., Sekido, Y., Kikuchi, S., Yasuda, I., Saheki, T., et al. (2001a). Novel mutations (H337R and 238-362del) in the CPS1 gene cause carbamoyl phosphate synthetase I deficiency. Hum. Hered. 52 (2), 99-101. doi: $10.1159 / 000053360$

Aoshima, T., Kajita, M., Sekido, Y., Mimura, S., Itakura, A., Yasuda, I., et al. (2001b). Carbamoyl phosphate synthetase I deficiency: molecular genetic findings and prenatal diagnosis. Prenat. Diagn. 21 (8), 634-637. doi: 10.1002/pd.123

Chen, M., Zhang, A., and Xu, Q. (2013). A case report of carbamoyl phosphate synthetase I deficiency. Jilin Med. (in Chinese) 34 (35), 7563-7564.

Chen, X., Yuan, L., Sun, M., Liu, Q., and Wu, Y. (2018). Two novel CPS1 mutations in a case of carbamoyl phosphate synthetase 1 deficiency causing hyperammonemia and leukodystrophy. J. Clin. Lab. Anal. 32 (5), e22375. doi: 10.1002/jcla.22375

Choi, R., Park, H. D., Yang, M., Ki, C. S., Lee, S. Y., Kim, J. W., et al. (2017). Novel pathogenic variant (c.580C > T) in the CPS1 gene in a newborn with carbamoyl phosphate synthetase 1 deficiency identified by whole exome sequencing. Ann. Lab. Med. 37 (1), 58-62. doi: 10.3343/alm.2017.37.1.58

de Cima, S., Polo, L. M., Díez-Fernández, C., Martínez, A. I., Cervera, J., Fita, I., et al. (2015). Structure of human carbamoyl phosphate synthetase: deciphering the on/off switch of human ureagenesis. Sci. Rep. 5, 16950. doi: 10.1038/ srep 16950

Diez-Fernandez, C., and Häberle, J. (2017). Targeting CPS1 in the treatment of carbamoyl phosphate synthetase 1 (CPS1) deficiency, a urea cycle disorder. Expert Opin. Ther. Targets 21 (4), 391-399. doi: 10.1080/14728222.2017. 1294685

Diez-Fernandez, C., Martínez, A. I., Pekkala, S., Barcelona, B., Perez-Arellano, I., Guadalajara, A. M., et al. (2013). Molecular characterization of carbamoylphosphate synthetase (CPS1) deficiency using human recombinant CPS1 as a key tool. Hum. Mutat. 34 (8), 1149-1159. doi: 10.1002/humu.22349

Diez-Fernandez, C., Hu, L., Cervera, J., Haberle, J., and Rubio, V. (2014). Understanding carbamoyl phosphate synthetase (CPS1) deficiency by using the recombinantly purified human enzyme: effects of CPS1 mutations that

\section{AUTHOR CONTRIBUTIONS}

This study was conceived and designed by ZG and YL. The experiments were conducted by KZ, MG, YQL, and HZ. Data analyzed by KZ and YL. BY, XL and ZG contributed clinical diagnosis of the patients. The paper was written by BY, CW and YL.

\section{ACKNOWLEDGMENTS}

This work was financially supported by Science and Technology Foundation of Shandong Province (2013GSF11829) and Jinan Excellent Science and Technology Innovation Team Project (20150515). The authors are grateful to the patients and their parents for their contribution to the study.

\section{SUPPLEMENTARY MATERIAL}

The Supplementary Material for this article can be found online at: https://www.frontiersin.org/articles/10.3389/fgene.2019.00718/ full\#supplementary-material

concentrate in a central domain of unknown function. Mol. Genet. Metab. 112 (2), 123-132. doi: 10.1016/j.ymgme.2014.04.003

Díez-Fernández, C., Gallego, J., Häberle, J., Cervera, J., and Rubio, V. (2015). The study of carbamoyl phosphate synthetase 1 deficiency sheds light on the mechanism for switching on/off the Urea Cycle. J. Genet. Genomics 42 (5), 249-260. doi: 10.1016/j.jgg.2015.03.009

Eeds, A. M., Hall, L. D., Yadav, M., Willis, A., Summar, S., Putnam, A., et al. (2006). The frequent observation of evidence for nonsense-mediated decay in RNA from patients with carbamyl phosphate synthetase I deficiency. Mol. Genet. Metab. 89 (1-2), 80-86. doi: 10.1016/j.ymgme.2006.04.006

Finckh, U., Kohlschuetter, A., Schaefer, H., Sperhake, K., Colombo, J. P., and Gal, A. (1998). Prenatal diagnosis of carbamoyl phosphate synthetase I deficiency by identification of a missense mutation in CPS1. Hum. Mutat. 12 (3), 206-211. doi: 10.1002/(SICI)1098-1004(1998)12:3<206::AID-HUMU8>3.0.CO;2-E

Funghini, S., Thusberg, J., Spada, M., Gasperini, S., Parini, R., Ventura, L., et al. (2012). Carbamoyl phosphate synthetase 1 deficiency in Italy: clinical and genetic findings in a heterogeneous cohort. Gene 493 (2), 228-234. doi: 10.1016/j.gene.2011.11.052

Hao, C., Guo, J., Guo, R., Qi, Z., Li, W., and Ni, X. (2018). Compound heterozygous variants in POR gene identified by whole-exome sequencing in a Chinese pedigree with cytochrome P450 oxidoreductase deficiency. Pediatr. Invest. 2 (2), 90-95. doi: 10.1002/ped4.12035

Häberle, J. (2012). Carglumic acid for the treatment of N-acetylglutamate synthase deficiency and acute hyperammonemia. Expert Rev. Endocrinol. Metab. 7 (3), 263-271. doi: 10.1586/eem.12.17

Häberle, J., Schmidt, E., Pauli, S., Rapp, B., Christensen, E., Wermuth, B., et al. (2003). Gene structure of human carbamylphosphate synthetase 1 and novel mutations in patients with neonatal onset. Hum. Mutat. 21 (4), 444. doi: 10.1002/humu.9118

Häberle, J., Shchelochkov, O. A., Wang, J., Katsonis, P., Hall, L., Reiss, S., et al. (2011). Molecular defects in human carbamoyl phosphate synthetase I: mutational spectrum, diagnostic and protein structure considerations. Hum. Mutat. 32 (6), 579-589. doi: 10.1002/humu.21406

Helman, G., Pacheco-Colon, I., and Gropman, A. L. (2014). The urea cycle disorders. Semin. Neurol. 34 (3), 341-349. doi: 10.1055/s-0034-1386771

Hoshide, R., Matsuura, T., Haraguchi, Y., Endo, F., Yoshinaga, M., and Matsuda, I. (1993). Carbamylphosphate synthetase I deficiency. One base substitution in an exon of the CPS1 gene causes a 9-basepair deletion due to aberrant splicing. J. Clin. Invest. 91 (5), 1884-1887. doi: 10.1172/JCI116405 
Ihara, K., Nakayama, H., Hikino, S., and Hara, T. (1999). Mutation in CPS1. Hum. Genet. 105, 375.

Janecková, H., Hron, K., Wojtowicz, P., Hlídková, E., Baresová, A., Friedecky, D., et al. (2012). Targeted metabolomics analysis of plasma samples for the diagnosis of inherited metabolic disorders. J. Chromatogr. A 1226, 11-17. doi: 10.1016/j. chroma.2011.09.074

Jia, J., and Shi, T. (2017). Towards efficiency in rare disease research: what is distinctive and important? Sci. China Life Sci. 60 (7), 686-691. doi: 10.1007/s11427-017-9099-3

Jia, J., An, Z., Ming, Y., Guo, Y., and Li, W. (2018). eRAM: encyclopedia of rare disease annotations for precision medicine. Nucleic Acids Res. 46, D937-D943. doi: 10.1093/nar/gkx1062

Jin, Y., Zhang, L., Ning, B., Hong, H., Xiao, W., Tong, W., et al. (2018). Application of genome analysis strategies in the clinical testing for pediatric diseases. Pediatr. Invest. 2 (2), 72-81. doi: 10.1002/ped4.12044

Khayat, M. (2009). Novel human pathological mutations. Gene symbol: CPS1. Disease: carbamoyl phosphate synthetase I deficiency. Hum. Genet. 125 (3), 336. doi: 10.1007/s00439-009-0642-9

Kretz, R., Hu, L., Wettstein, V., Leiteritz, D., and Häberle, J. (2012). Phytohemagglutinin stimulation of lymphocytes improves mutation analysis of carbamoylphosphate synthetase 1. Mol. Genet. Metab. 106 (3), 375-378. doi: 10.1016/j.ymgme.2012.04.011

Kurokawa, K., Yorifuji, T., Kawai, M., Momoi, T., Nagasaka, H., Takayanagi, M., et al. (2007). Molecular and clinical analyses of Japanese patients with carbamoylphosphate synthetase 1 (CPS1) deficiency. J. Hum. Genet. 52 (4), 349-354. doi: 10.1007/s10038-007-0122-9

László, A., Karsai, T., and Várkonyi, A. (1991). Congenital hyperammonemia: symptomatic carrier girl patient and her asymptomatic heterozygous mother for ornithine transcarbamylase (OTC) deficiency: specific enzyme diagnostic and kinetic investigations for the detection of heterozygous genostatus. Acta Paediatr. Hung. 31 (3), 291-297.

Lehotay, D. C., Hall, P., Lepage, J., Eichhorst, J. C., Etter, M. L., and Greenberg, C. R. (2011). LC-MS/MS progress in newborn screening. Clin. Biochem. 44 (1), 21-31. doi: 10.1016/j.clinbiochem.2010.08.007

Li, X., Yang, Y., Gao, Q., Gao, M., Lv, Y., Dong, R., et al. (2018). Clinical characteristics and mutation analysis of five Chinese patients with maple syrup urine disease. Metab. Brain Dis. 33 (3), 741-751. doi: 10.1007/s11011-017-0168-0

$\mathrm{Ni}$, X., and Shi, T. L. (2017). The Challenge and promise of rare disease diagnosis in China. Sci. China Life Sci. 60 (7), 681-685. doi: 10.1007/s11427-017-9100-1

Ono, H., Suto, T., Kinoshita, Y., Sakano, T., Furue, T., and Ohta, T. (2009). A case of carbamoyl phosphate synthetase 1 deficiency presenting symptoms at one month of age. Brain Dev. 31 (10), 779-781. doi: 10.1016/j.braindev.2008.12.013

Pekkala, S., Martinez, A. I., Barcelona, B., Yefimenko, I., Finckh, U., Rubio, V., et al. (2010). Understanding carbamoyl-phosphate synthetase I (CPS1) deficiency by using expression studies and structure-based analysis. Hum. Mutat. 31 (7), 801-808. doi: 10.1002/humu.21272

Raney, B. J., Dreszer, T. R., Barber, G. P., Clawson, H., Fujita, P. A., Wang, T., et al. (2014). Track data hubs enable visualization of user-defined genome-wide annotations on the UCSC Genome Browser. Bioinformatics 30, 1003-1005. doi: 10.1093/bioinformatics/btt637

Rapp, B., Häberle, J., Linnebank, M., Wermuth, B., Marquardt, T., Harms, E., et al. (2001). Genetic analysis of carbamoylphosphate synthetase I and ornithine transcarbamylase deficiency using fibroblasts. Eur. J. Pediatr. 160 (5), 283-287. doi: $10.1007 / s 004310100725$

Richards, S., Aziz, N., Bale, S., Bick, D., Das, S., Gastier-Foster, J., et al. (2015). Standards and guidelines for the interpretation of sequence variants: a joint consensus recommendation of the American College of Medical Genetics and Genomics and the Association for Molecular Pathology. Genet. Med. 17, 405424. doi: $10.1038 /$ gim. 2015.30

Rokicki, D., Pajdowska, M., Trubicka, J., Thong, M. K., Ciara, E., PiekutowskaAbramczuk, D., et al. (2017). 3-Methylglutaconic aciduria, frequent but underrecognized finding in carbamoyl phosphate synthetase I deficiency. Clin. Chim. Acta 471, 95-100. doi: 10.1016/j.cca.2017.05.023

Summar, M. L. (1998). Molecular genetic research into carbamoyl-phosphate synthase I: molecular defects and linkage markers. J. Inherit. Metab. Dis. 21 Suppl 1, 30-39. doi: 10.1023/A:1005349306311

Wakutani, Y., Nakayasu, H., Takeshima, T., Mori, N., Kobayashi, K., Endo, F, et al. (2001). A case of late-onset carbamoyl phosphate synthetase I deficiency, presenting periodic psychotic episodes coinciding with menstrual periods. Rinsho Shinkeigaku 41 (11), 780-785.

Wang, J., Shchelochkov, O. A., Zhan, H., Li, F., Chen, L. C., Brundage, E. K., et al. (2011). Molecular characterization of CPS1 deletions by array CGH. Mol. Genet. Metab. 102 (1), 103-106. doi: 10.1016/j.ymgme.2010.08.020

Wang, K., Li, M., and Hakonarson, H. (2010). ANNOVER: functional annotation of genetic variants from high-throughput sequencing data. Nucleic. Acids. Res. 38, e164. doi: 10.1093/nar/gkq603

Yang, X. Y., Shi, J., Lei, H. H., Xia, B., and Mu, D. Z. (2017). Neonatal-onset carbamoyl phosphate synthetase I deficiency. Medicine (Baltimore) 96 (26), e7365. doi: 10.1097/MD.0000000000007365

Zhang, Y., and Li, B. (2017). Cell and gene therapy for carbamoyl phosphate synthetase 1 deficiency. J. Pediatr. Neonatal Care 7 (1), 00273.

Zhang, G., Chen, Y., Ju, H., Bei, F., Li, J., Wang, J., et al. (2018). Carbamoyl phosphate synthetase 1 deficiency diagnosed by whole exome sequencing. J. Clin. Lab. Anal. 32 (2), e22241. doi: 10.1002/jcla.22241

Conflict of Interest Statement: The authors declare that the research was conducted in the absence of any commercial or financial relationships that could be construed as a potential conflict of interest.

Copyright (c) 2019 Yan, Wang, Zhang, Zhang, Gao, Lv, Li, Liu and Gai. The use, distribution or reproduction in other forums is permitted, provided the original author(s) and the copyright owner(s) are credited and that the original publication in this journal is cited, in accordance with accepted academic practice. No use, distribution or reproduction is permitted which does not comply with these terms. 